\title{
$X$. ON THE EXISTENCE OF CHRONIC PLAGUE IN RATS IN LOCALITIES WHERE PLAGUE IS ENDEMIC.
}

One of the most striking characteristics of plague, once it has become endemic in a locality, is its seasonal prevalence. At or about the same date it yearly reappears, rises, declines and disappears. In populous centres a few cases may occur throughout the year, but in smaller centres the disease commonly disappears entirely for at least six months.

The form in which the infection remains latent has been much speculated upon by epidemiologists, and it has been suggested by Simond (1898) and others that it may continue to exist in a chronic form among the rats. Kolle described chronic plague amongst rats in some of his laboratory experiments, but this form of plague among these animals has not, as far as we are aware, been observed in natural conditions. Tidswell failed to observe it in Sydney amongst the large number of examinations made in that city by the Board of Health during the years 1900 to 1904 .

In Bombay, where the Commission has been systematically examining both rats captured in traps and all dead rats discovered by the municipal "sweepers" throughout the year, and where large numberssometimes upwards of 5000-have been examined weekly, chronic plague has not so far been discovered, although plague in rats has been found to exist to a small extent throughout the year.

The existence of chronic plague in rats during the season when neither plague cases nor any acute plague amongst rats occurs has been systematically searched for by the Commission in the case of two villages, Kasel and Dhand, in the Punjab, in which plague has recurred annually for three years without discoverable reinfection, and which are the object of epidemiological study at the present time. Observations upon these villages commenced in December, 1905, at a time of year when neither human plague nor rat plague, as far as could be 
discovered, existed. There was no special mortality amongst the rats and dead rats were seldom found. An extensive rat-catching was undertaken with a view to finding out whether plague existed among these animals. During December 1800 rats were caught alive and carefully examined.

Of this number none were found to be suffering from ordinary acute plague, but on Dec. 12th, 1905 two, which until examined postmortem evinced no signs of illness, were discovered to have chronic abscesses. From these abscesses organisms morphologically indistinguishable from $B$. pestis were obtained, and after careful cultural and inoculation experiments no doubt remained that these rats which were runving about in apparent health were indeed suffering from chronic plague. During the following two weeks four more rats suffering from old abscesses were caught, and a fifth was caught in January, 1906. All of these abscesses proved to be due to chronic plague.

Case I. Mus rattus caught alive on 12. xii. 05 in Kasel.

Post-mortem examination showed nothing abnormal except an abscess in connection with the spleen. Numerous plague-like bacilli were found in smear preparations of the pus. Cultures on agar presented the appearances of a growth of $B$. pestis.

Animal Tests. (1) A guinea-pig was inoculated cutaneously with the pus of the abscess and died on 15. xii. 05. Exposure to cold was probably the cause of death. Nothing abnormal was seen on postmortem examination, and microscopical examination of the tissues proved to be negative.

(2) The agar culture from the abscess was inoculated cutaneously on the shaved abdomen of a guinea-pig on 13. xii. 05 ; this animal died on 21. xii. 05. P.-M.-The glands in the right and left inguinal and right axillary regions were enlarged and necrotic with surrounding haemorrhagic infiltration. The glands in the left axilla were congested. Grey nodules were present in the liver and there were numerous yellowish-grey nodules in the spleen; haemorrhages were seen under the capsule of the kidneys. The lungs were slightly congested and contained several large nodules similar to those in the liver. Preparations of the inguinal glands showed numerous plague-like organisms. The spleen showed a few clumps of organisms similar to those in the gland. The liver contained fairly numerous bacilli and the lung also numerous plague-like bacilli. A culture was made from this guineapig, subcultures of which gave typical stalactites in broth, furnished characteristic growths on agar and produced acid without gas in 
mannite, maltose, glucose and laevulose broths, but did not ferment lactose, cane-sugar nor dulcite. A rat inoculated subcutaneously with this culture on 20. i. 06 died on 22. i. 06 with typical appearances of plague. Smears from the inguinal gland, spleen and heart-blood showed numerous plague-like organisms.

Case II. Rat No. 754. Mus rattus caught alive on 12. xii. 05 in house 376, Kasel.

Post-mortem examination showed nothing abnormal except a small abscess in the lower end of the spleen. A few plague-like bacilli were found in smear preparations of the pus. Cultures of the pus were made on agar and gave growths resembling those of $B$. pestis. The stalactite test proved positive.

Animal Tests. (1) A culture from the abscess was inoculated cutaneously into a guinea-pig on 22. xii. 05. The animal developed marked infiltration at the site of inoculation and inguinal buboés, but recovered.

(2) A rat inoculated subcutaneously on 20 . i. 06 with two c.c. of a 48 hours' broth subculture (3rd remove) from the abscess died on 23. i. 06 with typical appearances of plague, e.g. marked subcutaneous congestion and granular liver. Preparations of the heart-blood and bubo showed many $B$. pestis and a spleen smear contained large numbers of plague bacilli.

Case III. Rat No. 1255. Caught alive on 18. xii. 05 in house No. 373, Dhand; female Mus rattus, weight 110 grams.

P.-M.-No emaciation or subcutaneous congestion. Nothing pathological was noted in the organs except the presence of numerous abscesses in the liver and mesentery. No bacilli were seen on microscopical examination of the organs and abscesses.

A culture was obtained from one of the abscesses which satisfied our tests of the plague bacillus, in that it gave typical stalactites, a characteristic growth on agar and reactions on sugar media similar to those previously referred to.

Case IV. Rat No. 1235. A Mus rattus trapped alive in house No. 407, Dhand, on 18. xii. 05.

P.M.-No emaciation or subcutaneous congestion. No secondary glands were noted, but one of the retroperitoneal (pelvic) glands was full of cheesy pus containing a few plague-like organisms; all the organs had a normal appearance. The heart-blood was not examined; the spleen showed no organisms. 
Cultures from the pus of the retroperitoneal gland were examined according to the methods already described; all the tests corresponded to those of the B. pestis.

Animal Test. A guinea-pig inoculated cutaneously with the pus from the retroperitoneal gland died on 23. xii. 05. P.-M.-A haemorrhagic infiltration surrounded the inguinal glands, which were necrotic on section. The liver contained a few very small granules, while the spleen was full of small greyish nodules. A few nodules were seen in the lungs; there was slight peritoneal effusion. Heart-blood showed very few bacilli; spleen and bubo, large numbers of $B$. pestis. Cultures from the organs were examined in detail and conformed completely to the tests of B. pestis. A rat inoculated with two c.c. of a 48 hours' subculture in broth from this guinea-pig on 20. i. 06 died on the night of 22. i. 06. P.-M.-Oedema at site of inoculation; liver mottled; smears from inguinal gland, spleen and heart-blood showed numerous plague-like organisms.

Case V. Rat No. 18321. M. rattus, caught in Dhaul village on 21. xii. 05 in the compound of a house in which a fatal case of plague had occurred about 11. xii. 05 and in which a convalescent case was discovered on 21. xii. 05. The rat was kept in a cage and died on 26. xii. 05 .

P.-M.-A small abscess containing cheesy pus was situated in the mesentery and was attached to the upper end of the spleen by a band of fibrous tissue. A smear from the spleen and from the pus showed no organisms.

A culture from the abscess proved to be plague by fulfilling the conditions already mentioned.

Animal Tests. (1) The pus was inoculated into the skin of a rat on 27. xii. 05. The rat was killed on 11. i. 06 ; post-mortem examination revealed nothing.

(2) Two c.c. of a 48 hours' broth culture (a subculture of the original) were inoculated subcutaneously into a rat on 20. i. 06 . The rat died on 22. i. 06. P.-M. - Slight oedema at site of inoculation; subcutaneous congestion; liver enlarged and congested. Smears from the liver, spleen, heart-blood and inguinal glands showed numerous plague-like organisms. Cultures from these tissues furnished plague-like growths.

Case VI. Rat No. 1779. Male Mus rattus; weight 105 grams; caught alive on 24. xii. 05 in house No. 623, Dhand.

1 This rat was not caught in the villages specially under examination but was brought in from a village-Dhaul in the neighbourhood. 
P.-M.-No emaciation nor subcutaneous congestion; no secondary glands but an abscess in the mesentery adherent to the spleen; organs normal in appearance; no organisms seen in heart-blood, spleen nor in pus of abscess.

Cultures were obtained from the pus of the abscess, which cultures resembled those of $B$. pestis, giving typical stalactites and the sugarreactions of the plague bacillus.

Animal Tests. (1) The pus from the abscess was inoculated cutaneously into a guinea-pig which died of plague on 30 . xii. 05 . P.-M.The inguinal glands on both sides were enlarged and necrotic with surrounding haemorrhagic effusion. The liver contained small areas of necrosis, and the spleen also was studded with yellowish-grey nodules. The lungs were congested and scattered through them were numerous yellowish nodules surrounded by hyperaemic tissue. There were haemorrhages in the suprarenal capsules. Heart-blood showed fairly numerous organisms; spleen and bubo large numbers of $B$. pestis. Cultures from this guinea-pig satisfied all the tests for B. pestis. A rat inoculated with two c.c. of a 48 hours' broth subculture on 20. i. 06 died on 22. i. 06. It showed oedema at the site of inoculation; the liver and spleen were granular. Smears from the spleen, liver, heartblood and inguinal glands showed numerous plague-like organisms.

(2) A rat inoculated with two c.c. of a 48 hours' broth subculture of the abscess on 20. i. 06 died on 23. i. 06. P.-M.-Marked subcutaneous congestion; oedema at site of injection. Smears of the spleen and of the heart's blood showed numerous plague-like organisms.

Case VII. Rat No. 2356. Mus rattus caught alive on 11. i. 06 in house No. 192, Dhand.

P.-M.-No emaciation nor subcutaneous congestion. One of the retroperitoneal (pelvic) glands was converted into an abscess; there was also a subcutaneous abscess in the right flank; all the organs appeared to be normal. Microscopically no organisms were observed in the pus of the retroperitoneal abscess. The subcutaneous abscess contained very numerous minute bacilli.

Cultures from the pelvic abscess furnished a growth resembling B. pestis, subcultures of which growth gave typical stalactites. No growth of plague could be recovered from the subcutaneous abscess.

Animal Test. The retroperitoneal abscess was inoculated cutaneously into a guinea-pig which died on 20. i. 06 . P.-M.-The inguinal glands on both sides were enlarged and necrotic with periglandular congestion and infiltration. The axillary glands on both sides were slightly enlarged. Numerous punctiform grey necrotic areas 
in liver; spleen studded with yellowish nodules; mesenteric vessels congested. Kidneys pale and mottled with subcapsular injection of blood-vessels. Numerous patches of congestion in lungs; slight pleural effusion. Heart-blood showed no organisms; spleen and bubo, large numbers of B. pestis. Cultures produced typical stalactites in broth.

\section{Chronic plague produced in rats by experimental inoculation.}

Out of 32 experimental rats which died of plague, presumably fleaborne (vide supra, pp. 445,9), two instances of chronic plague were found. In both instances the animals were killed 22 days after they had been first subjected to the chance of bites of infected fleas; they appeared quite healthy before they were destroyed and post-mortem examination showed all the organs to be healthy. In one instance there was an abscess in the right inguinal region, from the pus of which $B$. pestis was isolated. Subcultures of this organism killed rats with typical plague. In the other case there was a bubo in the right pelvis. As the animal was not examined till some time after death, the cultures taken were all contaminated, but subcultures from these, inoculated into rats, killed them with typical plague. It is apparent, therefore, that the organism in both these cases was still virulent.

\section{Summary.}

(1) It is a matter of considerable interest that, with the exception of rat No. 1832, the rats were caught alive during a period when no rats with acute plague were found and when no human cases were occurring in the locality.

(2) It cannot be regarded merely as a coincidence that the lesions were in every instance situated within the abdomen. With our present knowledge it would be rash to speculate as to the method of infection in these cases, but it may be conceded that the most reasonable interpretation of the lesions involves the assumption of an intestinal infection.

(3) Examination after the animals were killed furnished no indication that their health suffered in any way; the organs were apparently normal and there was no emaciation.

(4) Although the number of cases is admittedly small and although it is difficult to estimate virulence with any degree of accuracy, yet it is noteworthy that of the animals inoculated directly from, and with subcultures of, these abscesses only one survived. 


\section{TABLE.}

Situation of lesion

Abscess in spleen

Abscess in spleen

Multiple abscesses in liver and mesentery

\section{Retroperitoneal abscess}

Mesenteric abscess

Mesenteric abscess
Microscopical examination of pus

Numerous bacilli like B. pestis
A few plague-like bacilli
all positive

No bacilli seen

A few plague-like bucilli

No bacilli seen

\section{Cultural tests}

like $B$. pestis on agar (not examined further)

,

,

9

,

, abscess

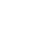

Culture from abscess inoculated cutaneou pig, which died in 8 days of plague

Rat inoculated suboutaneously with cultur pig died acutely of plague

Culture from abscess inoculated cutaneou pig-recovered after local reaction and $k$

Rat; subculture from abscess subca oculated-died acutely of plague

\section{None made}

Pus; cutaneous inoculation; guinea-pig i of plague

Rat; subcutaneously inoculated; culture pig; died acutely of plague

Pus; cutaneous inoculation; rat; negativ Subculture pus; subcutaneous inoculat acutely of plague

Pus; cutaneous inoculation; guinea-pig in 6 days

Culture from guinea-pig; subcutaneous ir died of plague acutely

Subculture from abscess; subcutaneous ir died of plague acutely

Pus; cutaneous inoculation; guinea-pig । in 9 days 